\title{
The algorithm for the partition function of the generalised Ising model on a square lattice
}

\author{
P. Kozłowski, G. Kamieniarz, G. Musiał
}

\author{
Computational Physics Division, \\ Institute of Physics, A. Mickiewicz University, \\ ul. Umultowska 85, 61-614 Poznań, Poland \\ e-mail: gjk@pearl.amu.edu.pl
}

\begin{abstract}
The paper presents the algorithm and description of a procedure gim written in the FORTRAN 90 language, for calculation of the partition function of the generalised Ising model on a square lattice. The procedure is available at the Poznań Supercomputing and Networking Center on a Cray J916 supercomputer in numerical procedure library mestat.
\end{abstract}

\section{Introduction}

Spontaneous ordering of atomic spins is one of the most interesting phenomena in solid state physics. Unfortunately, exact quantitative description of ferro- and antiferromagnetism is not possible in terms of the existing theories, therefore considerations are performed on models of these phenomena among which particularly important is the Ising model [1], The main advantage of this model is the exact solvability of its two-dimensional version which is a non-trivial example of a system in which magnetic phase transitions can be analysed exactly.

In the Ising model only one order parameter is considered. A natural extension of the Ising model is the Ashkin-Teller model [2] which is based on three order parameters and two kinds of spin variables.

In this model a set of $N$ fixed points, called the lattice sites, forming a 2 -dimensional periodic lattice of regular structure, is considered. Each site is attributed with a spin variable (henceforth denoted as $S_{i}$ or $\sigma_{i}, 2=1,2, \ldots, N$ ) 
which is a number and can take values of +1 or -1 . The system has no other degrees of freedom.

The partition function is defined by the expression:

$$
\mathcal{Z}=\sum \mathrm{e}^{-\beta H}
$$

where the summation runs over all configurations of spin variables 5 and $\sigma, H$ is the hamiltonian of the system, $\beta=1 / k_{\mathrm{B}} T$ is the inverse of the product of temperature $T$ and the Boltzmann constant $k_{B}$. The partition function $\mathcal{Z}$ is one of the fundamental quantities in the statistical physics as the logarithm of the partition function multiplied by $-\beta^{-1}$ gives the free energy of the system which is necessary to obtain the thermodynamic functions of the system.

The gim procedure was written for a planar square lattice and permits a calculation of the partition function $\mathcal{Z}$ for the Ising model per a single lattice site, taking advantage of the accurate algorithm based on the transfer matrix. Given the actual values of appropriate parameters, the procedure enables the calculations for the Ashkin-Teller or standard Ising models.

\section{The Hamiltonian and the algorithm of calculations}

The starting point for the calculations is the Hamiltonian of the system which for the Ashkin-Teller model takes the form:

$$
\mathcal{H}=-\beta H=-\mathrm{pk} 1 \sum_{\langle i, j\rangle} S_{i} S_{j}-\mathrm{pk} 2 \sum_{\langle i, j\rangle} \sigma_{i} \sigma_{j}-\mathrm{pk} 4 \sum_{\langle i, j\rangle} S_{i} \sigma_{i} S_{j} \sigma_{j},
$$

where the summation runs over pairs of the nearest neighbours on an $L \times 2$ size square lattice with periodic boundary conditions, and pkl, pk2, pk4 are the reduced coupling constants which as the parameters of the procedure are written down by thick letters.

The hamiltonian of the standard Ising model is a particular case of hamiltonian (2) restricted to the first term:

$$
\mathcal{H}=-\operatorname{pk} 1 \sum_{\langle i, j\rangle} S_{i} S_{j}
$$

In this model there is only one kind of spin variable.

To calculate the partition function we make use of the perturbation transfer matrix method [3] and the following relation [4]:

$$
\mathcal{Z}_{0}=\sum_{n}\left\langle n\left|\mathbf{T}^{L}\right| n\right\rangle
$$


where $L$ is the number of sites in a column or a line, $\mathbf{T}$ is the transfer matrix, and $|n\rangle$ vectors form a $4^{L}$ dimensional base of the transfer matrix $\mathbf{T}$ of the AshkinTeller model and $2^{L}$ dimensional base of the Ising model. The smaller base for the latter model permits calculations for larger systems.

The memory required to carry out this algorithm was minimised having recourse to the fact that only two $|n\rangle$ vectors are needed for the calculations. The algorithm is based on the numerically exact calculations of the trace (4) where inaccuracies are only due to round-ups. It is estimated that about 12 significant digits of the result are accurate.

\section{Description of the gim procedure}

The gim procedure is written in FORTRAN 90 with the use of a vectorizable algorithm.

The call of the procedure:

$$
\text { call } \operatorname{gim}(1, \mathrm{pkl}, \mathrm{pk2}, \mathrm{pk4}, \mathrm{zO}, \mathrm{w})
$$

The input parameters:

1 - an integer type variable which determines the number of sites in a line (column) and can take the values: $2,3, \ldots, M_{\max }$, where $M_{\max }$ is 10 and 20 for the Ashkin-Teller and Ising model, respectively.

pkl,pk2,pk4 - are real*8 type variables determining the values of the hamiltonian (2) parameters for the Ashkin-Teller model, whereas for the Ising model pkl determines the value of the hamiltonian (3) parameter.

w - a character* 1 type variable whose value determines for which model the calculations are performed, i.e. when it takes a value $I$ or $i$ the calculations are for the standard Ising model, otherwise for the Ashkin-Teller model.

The output parameters:

zO - a real*8 type variable which contains the partition function in the zero field. It is the partition function per a single site of a 1 x 1 size lattice.

\section{Acknowledgements}

The authors wish to acknowledge the access to Cray J916 supercomputer at the Poznań Supercomputing and Networking Center and partial financial support from the Committee for Scientific Research within the grant 8T11F 01509. 
References

[1] E. Ising, Z. Phys. 31, 253 (1925);

C.N. Yang, Phys. Rev. 85, 808 (1952)

[2] J. Askhin and E. Teller, Phys. Rev. 64, 178 (1943)

[3] G. Kamieniarz, H.W.J. Blöte, J. Phys. A26, 201 (1993)

[4] G. Kamieniarz, P. Kozłowski, R. Dekeyser, Phys. Rev. E55, 3724 (1997) 